\title{
Electronic Properties of Topological Materials: Optical Excitations in Möbius Conjugated Polymers
}

\author{
Kikuo Harigaya* \\ Nanotechnology Research Institute, AIST, Tsukuba 305-8568, Japan ${ }^{\dagger}$ \\ Synthetic Nano-Function Materials Project, AIST, Tsukuba 305-8568, Japan \\ (Received
}

\begin{abstract}
Electronic structures and optical excitations in Möbius conjugated polymers are studied theoretically. Periodic and Möbius boundary conditions are applied to the tight binding model of poly(para-phenylene), taking exciton effects into account. We discuss that oligomers with a few structural units are more effective than polymers for observations of effects of discrete wave numbers that are shifted by the change in boundary condition. Next, calculations of optical absorption spectra are reported. Certain components of optical absorption for an electric field perpendicular to the polymer axis mix with absorption spectra for an electric field parallel to the polymer axis. Therefore, the polarization dependences of an electric field of light enable us to detect whether conjugated polymers have the Möbius boundary.
\end{abstract}

KEYWORDS: Möbius conjugated polymers, topological materials, optical excitations, theory

\footnotetext{
*E-mail address: k.harigaya@aist.go.jp; URL: http://staff.aist.go.jp/k.harigaya/

${ }^{\dagger}$ Corresponding address
} 
Recently, low-dimensional materials with peculiar boundary conditions, i.e., Möbius boundaries have been synthesized: they are $\mathrm{NbSe}_{3}$ (ref. 1) and aromatic hydrocarbons ${ }^{2}$, for example. A Möbius strip consists of one surface that does not have the difference between the outer and inner surfaces. The orbitals of electrons are twisted while traveling along the strip axis, and electronic states can be treated with antiperiodic boundary conditions mathematically. Even though the presence of twisted $\pi$-electron systems has been predicted theoretically about forty years ago, ${ }^{3)}$ structural perturbations due to topological characters might result in physical properties, thus intensive investigations are being performed experimentally and theoretically.

In a theoretical viewpoint, we have studied boundary condition effects in nanographite systems where carbon atoms are arrayed in a one-dimensional shape with zigzag edges. ${ }^{4-6)}$ Due to the presence of a Möbius boundary, magnetic domain wall states and helical magnetic orders are realized in spin alignments, and a domain wall also appears in charge density wave states. Such abundant properties have been experimentally observed by their unique magnetic properties. $^{7,8)}$

In this paper, we investigate another candidate for twisted $\pi$-electron systems: conjugated polymers. We choose poly(para-phenylene) (PPP) as a model material of conjugated polymers. The structure of PPP is shown in Fig. 1. Optical excitations in periodic systems have been previously studied using a tight binding model with long-range Coulomb interactions for poly (para-phenylenevinylene) $(\mathrm{PPV}),{ }^{9)} \mathrm{PPP}$, and so forth. ${ }^{10)}$ Exciton effects have been taken into account by the configuration interaction method. Here, we study optical excitations in Möbius PPP strips. We expect that such predictive studies of electronic properties will promote the synthesis of materials with unique geometries.

We will discuss that oligomers with a few structural units are more effective than polymers for measurements of effects of discrete wave numbers that are shifted from those of the periodic boundary by the Möbius boundary. When ring-shaped oligomers ${ }^{11,12)}$ are converted into Möbius oligomers, such effects could be observed by experiments. Next we will calculate optical absorption spectra for the periodic [Fig. 2(a)], Möbius [Figs. 2(b) and 2(c)] PPP strips. We 
consider two cases of geometries for the Möbius strips: (I) the twist of the bonds is spatially uniform, as shown schematically in Fig. 2(b), and (II) twist positions are spatially limited with respect to the dimensions of the entire strip, as shown in Fig. 2(c). We will reveal that certain components of optical absorption for an electric field perpendicular to the polymer axis mix with absorption spectra for an electric field parallel to the polymer axis. The polarization dependences of an electric field of light enable us to detect whether conjugated polymers have the Möbius boundary. The dependences on the geometries of the Möbius strips are also reported.

We consider optical excitations in PPP using a model ${ }^{9)}$ with electron-phonon and electronelectron interactions. The model is shown below:

$$
\begin{aligned}
H & =H_{\mathrm{pol}}+H_{\mathrm{int}}, \\
H_{\mathrm{pol}} & =-\sum_{\langle i, j\rangle, \sigma}\left(t-\alpha y_{i, j}\right)\left(c_{i, \sigma}^{\dagger} c_{j, \sigma}+\text { h.c. }\right)+\frac{K}{2} \sum_{\langle i, j\rangle} y_{i, j}^{2}, \\
H_{\mathrm{int}} & =U \sum_{i}\left(c_{i, \uparrow}^{\dagger} c_{i, \uparrow}-\frac{n_{\mathrm{el}}}{2}\right)\left(c_{i, \downarrow}^{\dagger} c_{i, \downarrow}-\frac{n_{\mathrm{el}}}{2}\right) \\
& +\sum_{i, j} W\left(r_{i, j}\right)\left(\sum_{\sigma} c_{i, \sigma}^{\dagger} c_{i, \sigma}-n_{\mathrm{el}}\right)\left(\sum_{\tau} c_{j, \tau}^{\dagger} c_{j, \tau}-n_{\mathrm{el}}\right) .
\end{aligned}
$$

In eq. (1), the first term $H_{\text {pol }}$ is the tight binding model along the polymer backbone with electron-phonon interactions, which couple $\pi$-electrons with modulation modes of bond lengths, and the second term $H_{\text {int }}$ is the Coulomb interaction potential among $\pi$-electrons. In eq. (2), $t(>0)$ is the hopping integral between the nearest neighbor $i$ th and $j$ th sites in an ideal system without bond alternations; $\alpha$ is the electron-phonon coupling constant that modulates the hopping integral linearly with respect to the bond variable $y_{i, j}$ that measures the magnitude of the alternation of the bond $\langle i, j\rangle ; y_{i, j}>0$ for long bonds and $y_{i, j}<0$ for short bonds (the average of $y_{i, j}$ is taken to be zero); $K$ is the harmonic spring constant for $y_{i, j}$; and the sum is taken over pairs of neighboring atoms. Equation (3) indicates the Coulomb interaction among electrons. Here, $n_{\mathrm{el}}$ is the average number of electrons per site; $r_{i, j}$ is the distance between the $i$ th and $j$ th sites; and $W(r)=1 / \sqrt{(1 / U)^{2}+(r / a V)^{2}}$ is the parametrized Ohno potential. The quantity $W(0)=U$ is the strength of the on-site interaction; $V$ is the strength of the long-range part $(W(r) \sim a V / r$ in the limit $r \gg a)$; and $a$ is the mean bond length. 
Electron-phonon interactions are treated by classical approximation with complete lattice relaxation as in ref. 9. Excitation wave functions of an electron-hole pair are calculated by the Hartree-Fock approximation followed by the single-excitation configuration interaction method. This method, which is appropriate for cases of moderate Coulomb interactions, that is, strengths between negligible and strong Coulomb interactions, is known as the intermediate exciton theory used in ref. 9. We write singlet electron-hole excitations as $|\mu, \lambda\rangle=(1 / \sqrt{2})\left(c_{\mu, \uparrow}^{\dagger} c_{\lambda, \uparrow}-c_{\mu, \downarrow}^{\dagger} c_{\lambda, \downarrow}\right)|g\rangle$, where $\mu$ and $\lambda$ are unoccupied and occupied states, respectively, and $|g\rangle$ is the Hartree-Fock ground state. The general expression of the $\kappa$ th optical excitation is $|\kappa\rangle=\sum_{(\mu, \lambda)} D_{\kappa,(\mu, \lambda)}|\mu, \lambda\rangle$. The optical absorption spectrum $\alpha(\omega)$ is calculated using these excitations for $l=x, y, z$ polarizations: $\alpha(\omega)=\sum_{\kappa} E_{\kappa} L\left(\omega-E_{\kappa}\right)\langle g|l| \kappa\rangle\langle\kappa|l| g\rangle$, where $E_{\kappa}$ is the optical excitation energy of the state $|\kappa\rangle$ and $L(\omega)$ is the Lorentzian function $L(\omega)=\gamma /\left[\pi\left(\omega^{2}+\gamma^{2}\right)\right]$.

The difference between the periodic and Möbius boundary conditions is regarded as due to the addition of antiperiodicity. As well known in the textbook of condensed matter physics, the allowed wave numbers are different between periodic and antiperiodic boundary conditions. Because the allowed states in the wave number space are densely populated in a sufficiently long polymer, the effects of the boundary condition difference are too small to be observed. However, in oligomers with a few phenyl rings, the allowed states are so sparsely distributed that the boundary condition effects could be measured. The recent synthesis of Möbius aromatic systems $^{2)}$ will promote the synthesis of Möbius polymers in view of the presence of many types of ring polymers. ${ }^{11,12)}$

In the following, we discuss the selection rules for the PPP case. The electronic states in the wave number space are shown for the number of phenyls $N=5$ in Fig. 3. The electronic states of the tight binding model with the hopping integral $t$ only are shown. The allowed states for the periodic and antiperiodic boundary conditions are shown by filled and open circles, respectively. The allowed wave numbers are $k=j(2 \pi / b N)$ for $j=0$ to $N-1$ in the extended zone scheme for the periodic boundary condition, $b$ being the size of the unit cell. They are $k=(j+1 / 2)(2 \pi / b N)$ for $j=0$ to $N-1$ in the Möbius (antiperiodic) boundary condition. If 
$N$ is of the orders of a few, the allowed wave numbers are sparsely distributed. The change in the lowest optical transition energy around the $\Gamma$ point between the two boundary conditions is expected. However, the distribution of the allowed wave numbers becomes dense as $N$ increases. Therefore, the experimental synthesis of the Möbius oligomers with a few phenyl rings will be necessary for measuring the change of electronic structures from those of the periodic oligomers.

First, we discuss the optical absorption of PPP for the periodic boundary condition. As in ref. 9, we use the following parameters: $\alpha=2.59 t / \AA, K=26.6 t / \AA^{2}, U=2.5 t$, and $V=1.3 t$, where the parameters of the dimensions of energies are shown in the unit of $t$. The $t$ actual value is $2.3 \mathrm{eV}$ for PPV (ref. 9). Similar magnitudes are also expected for PPP. The number of phenyl rings is $N=20$. Figure $2($ a) shows the geometry of the strip with a periodic PPP chain. The circle of the polymer axis is assumed to be placed within the $x-y$ plane. The $z$-axis is perpendicular to the polymer axis. Figure 4 shows the calculated absorption with the Lorentzian broadening $\gamma=0.15 t$. The unit of the vertical axis is taken to be arbitrary.

When an electric field of light is parallel to the polymer axis [Fig. 4(a)], the lowest optical excitations appear at an energy of about 1.4t. They are excitations between the extended states, which are the states at the top of the valence band and those at the bottom of the conduction band in Fig. 3. Off course, the excitation energies calculated by taking exciton effects into account are different from those in Fig. 3, where the electronic states of the free electron model are displayed. The higher feature at $2.4 t$ is due to the optical excitations between the localized occupied and unoccupied orbitals. The localized orbitals are shown in Fig. 3. When the electric field is perpendicular to the polymer axis [Fig. 4(b)], the optical excitations appear at energies larger than 2.2t. They are excitations between the extended states and the localized orbitals. Figure 4(c) shows the averaged spectrum with respect to the polarization of light, i.e., the sum over $x-, y$-, and $z$-polarizations. The overall features have been observed experimentally. For details, refer to previously published reports. ${ }^{9,10)}$ In energies higher than $\sim 3 t$, optical excitations including $\sigma$-electrons mix with absorption spectra. Such effects require calculations with both $\pi$ - and $\sigma$-electrons. 
Next, we consider two Möbius boundary cases. The first case (I) is that ring torsions uniformly occur over a polymer with the torsion angle $\Psi=180^{\circ} / N$. Phenyl rings helically rotate along with the polymer. Figure 2(b) shows the geometry of the polymer strip. The polymer axis is represented by a circle in the $x-y$ plane as in the periodic boundary case. The plane of the PPP strip is along the $x$-axis where $x>0$ and the polymer crosses the $x$-axis, and the polymer plane is perpendicular to the $x$-axis where $x<0$. Where the polymer strip crosses the $y$-axis, the polymer plane leans by $45^{\circ}$ within the $y$ - $z$ plane. The second case (II) is that the twist due to the Möbius boundary is localized among five phenyl rings where the polymer circle crosses the $x$-axis. The geometry is shown in Fig. 2(c). The twist angle from the torsion is taken to be $\Psi=30^{\circ}$ as a representative value. The modulation of the hopping integral by the torsion is taken into account by the replacement $t-\alpha y \Rightarrow(t-\alpha y) \cos \Psi$, at the bonds where torsions are present. Hereafter, the calculated absorption spectra are shown for the number of phenyl rings $N=20$. The uniform torsion of about $23^{\circ}$ is not considered, because the modulation of the overall shape of the optical spectra is small even if we consider the uniform torsion to be zero. ${ }^{10)}$

In case I, the plane that includes the polymer is almost parallel to the $x-y$ plane in a certain part of the polymer. This occurs near the region where the polymer crosses the positive part of the $x$-axis [Fig. 2(b)]. In this part, an almost perpendicular polarization is realized, and therefore mixing of the absorption due to this perpendicular polarization [Fig. 4(b)] is expected. Figure 5(a) shows the actual calculation. There are two weak features due to the mixing among the energies $2.2 t$ and 3.0t. There is no difference between the $x$ - and $y$-polarizations, because the system is uniform. On the other hand, the absorption of the perpendicular polarization does not show any polarization dependence [Fig. 5(b)].

In case II, the polymer plane can become parallel to the $x-y$ plane at the region where the twist is present. This is the part where the polymer strip meets the positive region of the $x$-axis [Fig. 2(c)]. There is a nearly perpendicular polarization in this region. The mixing of the perpendicular polarization occurs among the energies 3.0t as shown in Fig. 6(a), where the 
electric field of light is parallel to the $x$-axis. For the case that the field is along the $y$-axis, the mixing of the perpendicular polarization is very weak because the presence of twists is spatially limited in the entire geometry of the polymer chain [Fig. 6(b)].

For the fixed helical torsion angle $\Psi=30^{\circ}$ and $N=6$, cases I and II are equivalent. As $N$ increases, differences between the two Möbius structures are expected to be more apparent. Therefore, the selection rule difference in the wave number space will be easier to be observed for short oligomers. On the other hand, structure-dependent optical absorption will reflect what types of Möbius rings are formed in more long polymers or oligomers $(N>10)$.

In summary, electronic structures and optical excitations in PPP with periodic and Möbius boundaries have been studied by taking exciton effects into account. In the calculated optical absorption spectra, certain components of optical absorption for an electric field perpendicular to the polymer axis mix with absorption spectra for an electric field parallel to the polymer axis. The polarization dependences of an electric field of light enables us to detect whether conjugated polymers have the Möbius boundary. Therefore, the experimental synthesis of materials with unique geometries is expected.

\section{Acknowledgments}

This work has been supported partly by Special Coordination Funds for Promoting Science and Technology, and by NEDO under the Nanotechnology Program. 


\section{References}

1) S. Tanda et al.: Nature 417 (2002) 397.

2) D. Ajami et al.: Nature 426 (2003) 819.

3) E. Heilbronner: Tetrahedron Lett. 5 (1964) 1923.

4) K. Wakabayashi and K. Harigaya: J. Phys. Soc. Jpn. 72 (2003) 998.

5) A. Yamashiro, Y. Shimoi, K. Harigaya and K. Wakabayashi: Phys. Rev. B 68 (2003) 193410.

6) A. Yamashiro, Y. Shimoi, K. Harigaya and K. Wakabayashi: Physica E 22 (2004) 688.

7) Y. Shibayama et al.: Phys. Rev. Lett. 84 (2000) 1744.

8) H. Sato et al.: Solid State Commun. 125 (2003) 641.

9) K. Harigaya: J. Phys. Soc. Jpn. 66 (1997) 1272.

10) K. Harigaya: J. Phys.: Condens. Matter 10 (1998) 7679.

11) E. Mena-Osteritz: Adv. Mater. 14 (2002) 609.

12) M. Mayor and C. Didschies: Angew. Chem. Int. Ed. 42 (2003) 3176. 


\section{Figure Captions}

Fig. 1. Polymer structures of poly(para-phenylene) (PPP).

Fig. 2. Geometries of PPP strips used for calculations: (a) periodic polymer, (b) spatially uniform Möbius polymer, and (c) Möbius polymer with spatially localized twists. The PPP chain is represented schematically by the strip. The $\pi$-orbitals of carbons extend perpendicularly with respect to the strip.

Fig. 3. Electronic states of PPP in wave number space, shown for phenyl ring number $N=5$. The case of the simple tight binding model with the hopping integral $t$ is shown. The allowed states for the periodic and antiperiodic boundary conditions are shown by filled and open circles, respectively. The solid lines represent the electronic states of the polymer with an infinite length.

Fig. 4. Optical absorption spectra for periodic boundary case: (a) electric field of light parallel to polymer axis, (b) electric field perpendicular to polymer axis, and (c) nonpolarized absorption. The number of phenyl rings is $N=20$. The vertical axis is shown in arbitrary units, and the Lorentzian broadening $\gamma=0.15 t$ is used in the calculations.

Fig. 5. Optical absorption spectra for Möbius boundary case I: (a) electric field of light parallel to polymer axis and (b) electric field perpendicular to polymer axis. The circles show features due to the mixing of perpendicular polarization. The number of phenyl rings is $N=20$. The vertical axis is shown in arbitrary units, and the Lorentzian broadening $\gamma=0.15 t$ is used in the calculations.

Fig. 6. Optical absorption spectra for Möbius boundary case II: (a) electric field of light parallel 
to $x$-axis and (b) electric field of light parallel to $y$-axis. The circles show features due to the mixing of perpendicular polarization. The number of phenyl rings is $N=20$. The vertical axis is shown in arbitrary units, and the Lorentzian broadening $\gamma=0.15 t$ is used in the calculations. 
PPP: poly(para-phenylene)

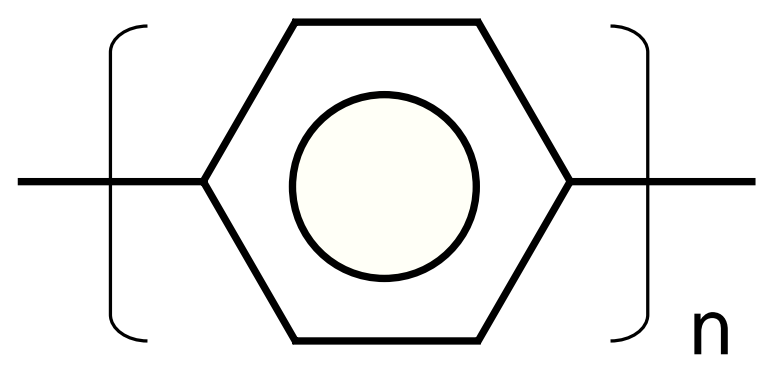

Fig. 1 

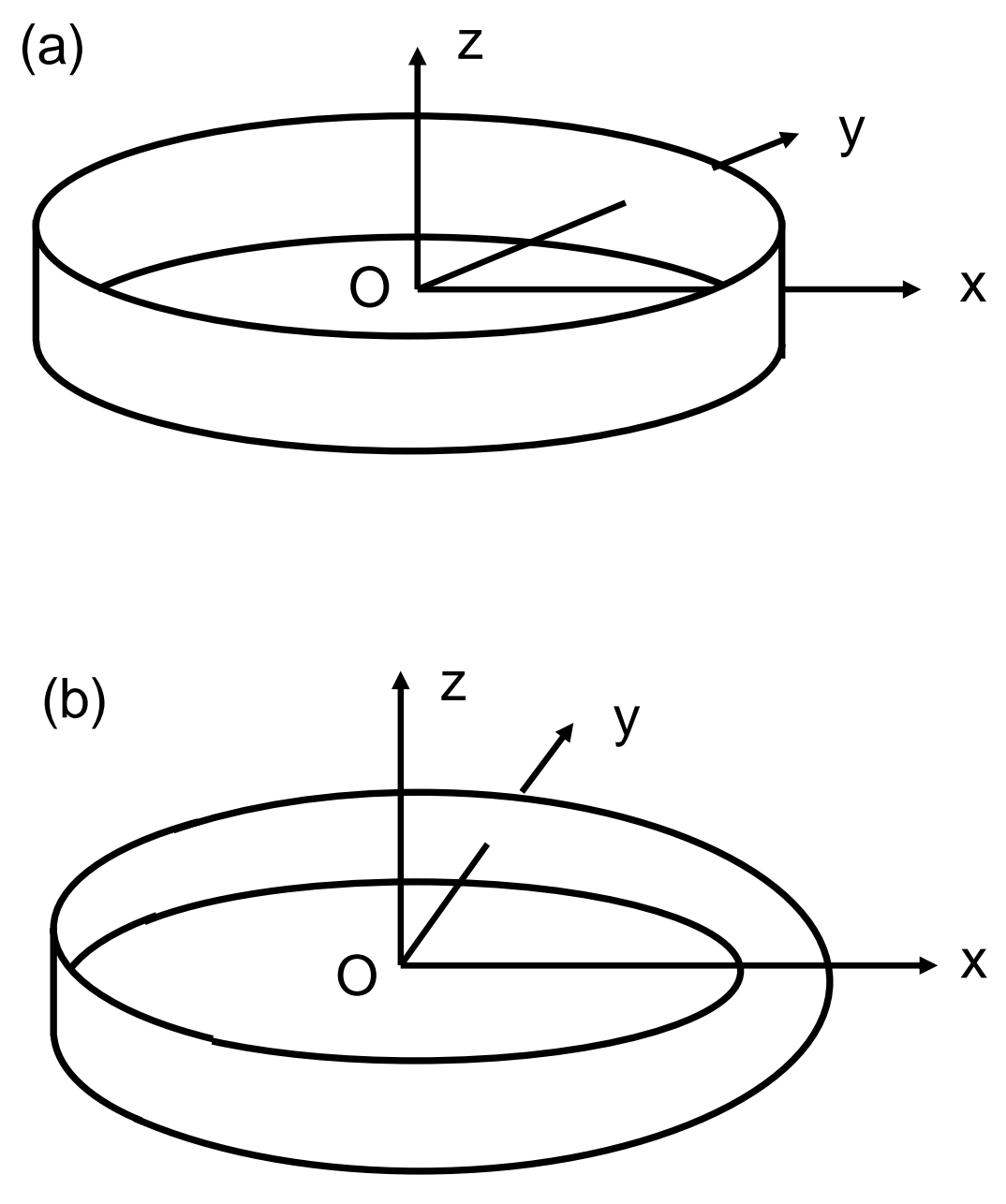

Fig. 2 


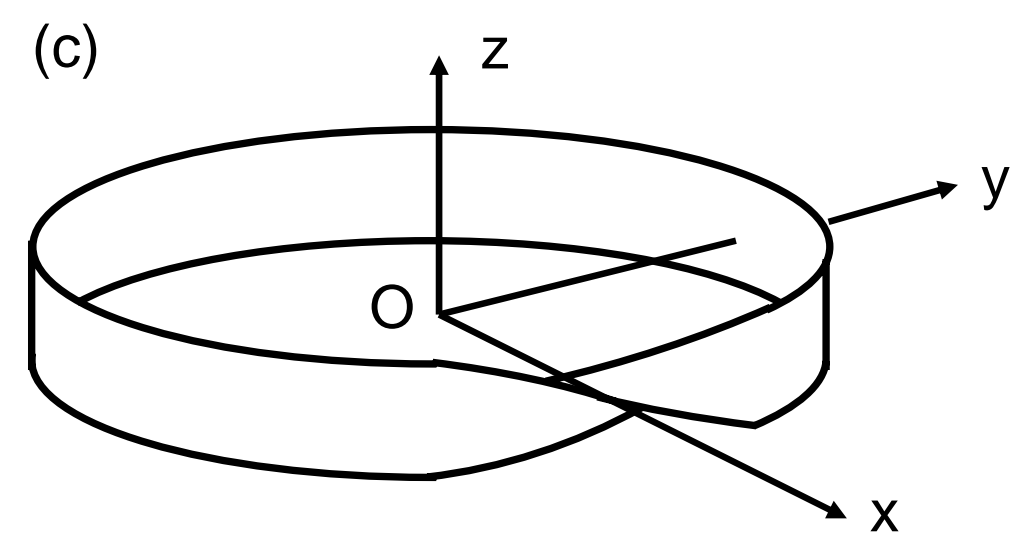

Fig. 2 


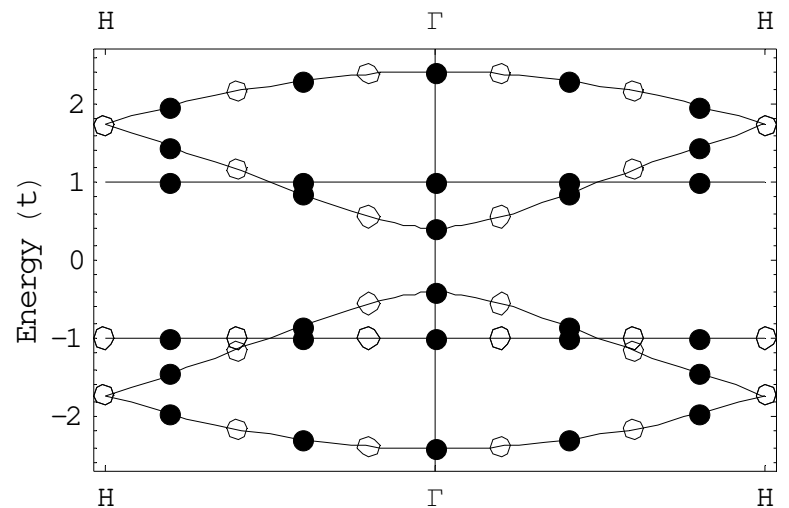

Fig. 3 

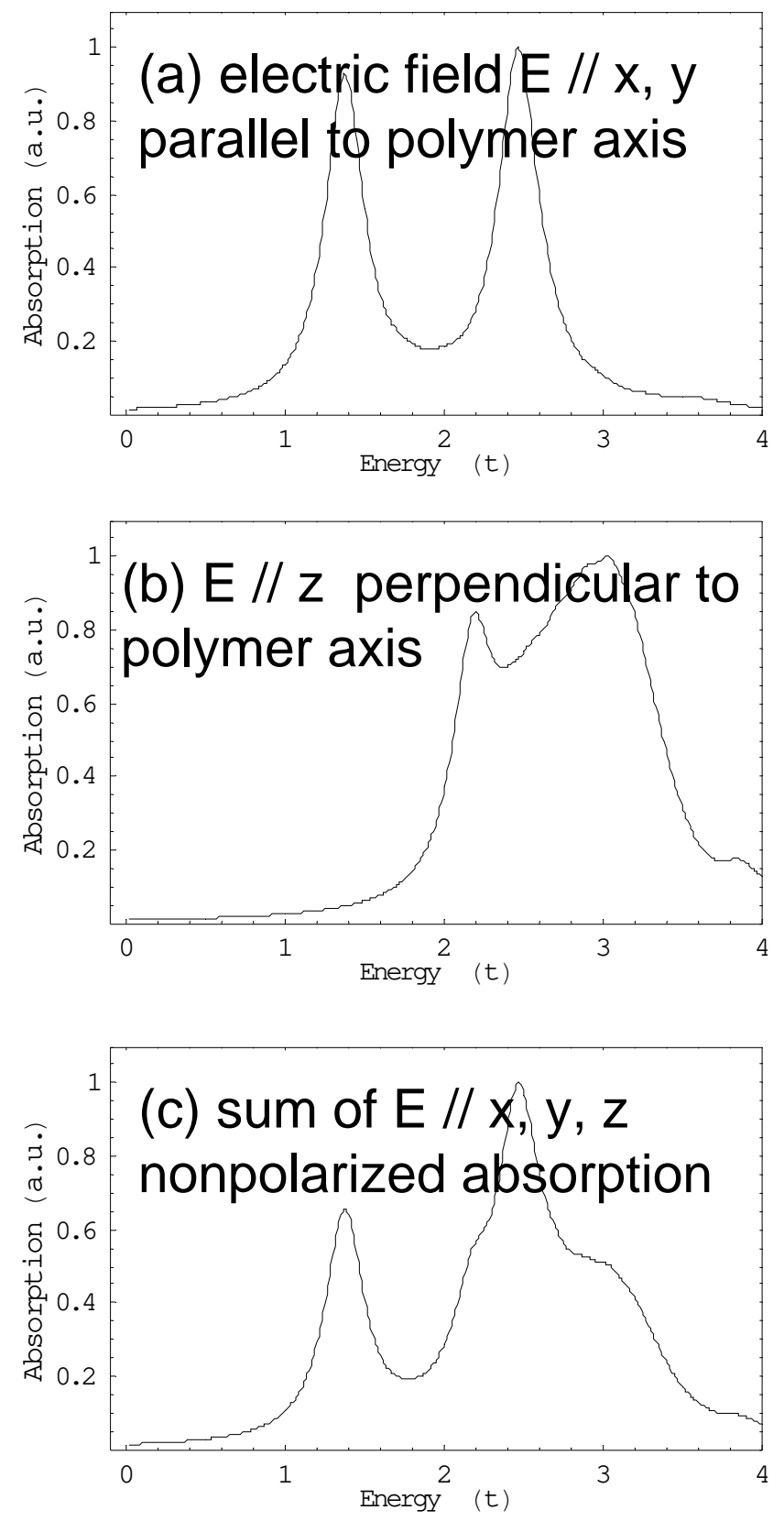

Fig. 4 

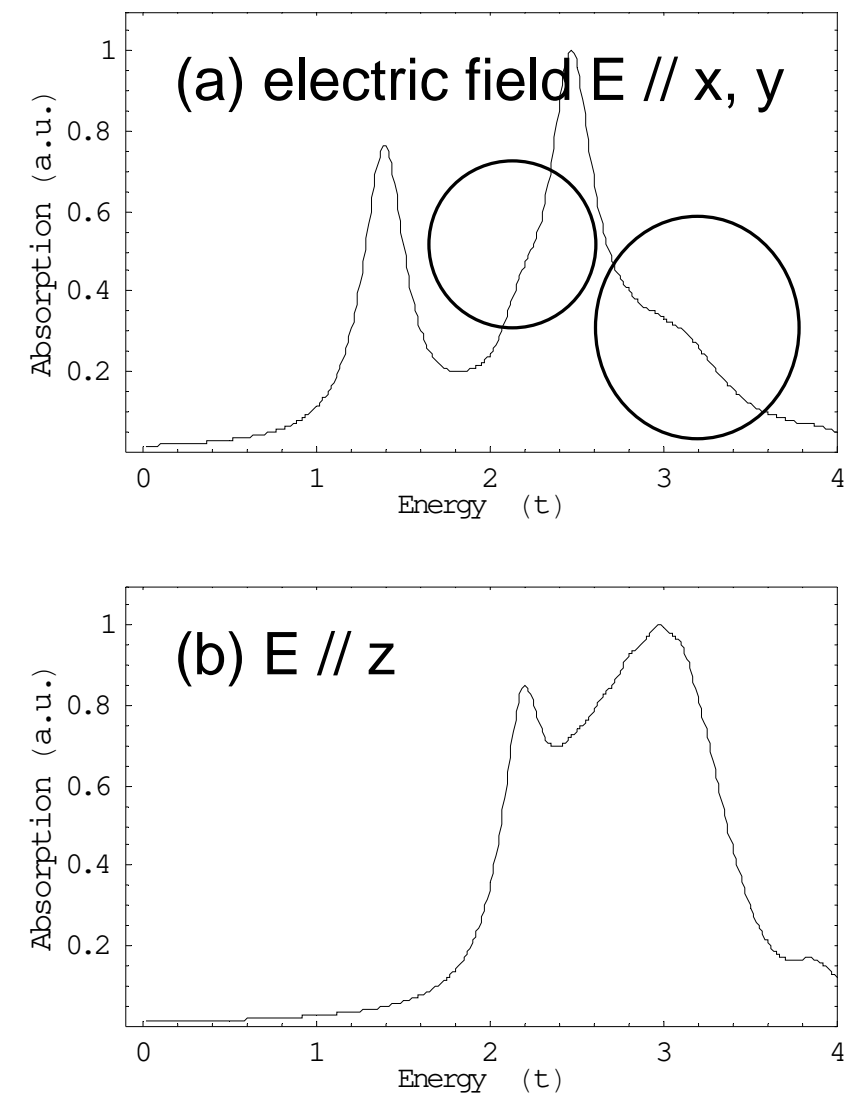

Fig. 5 

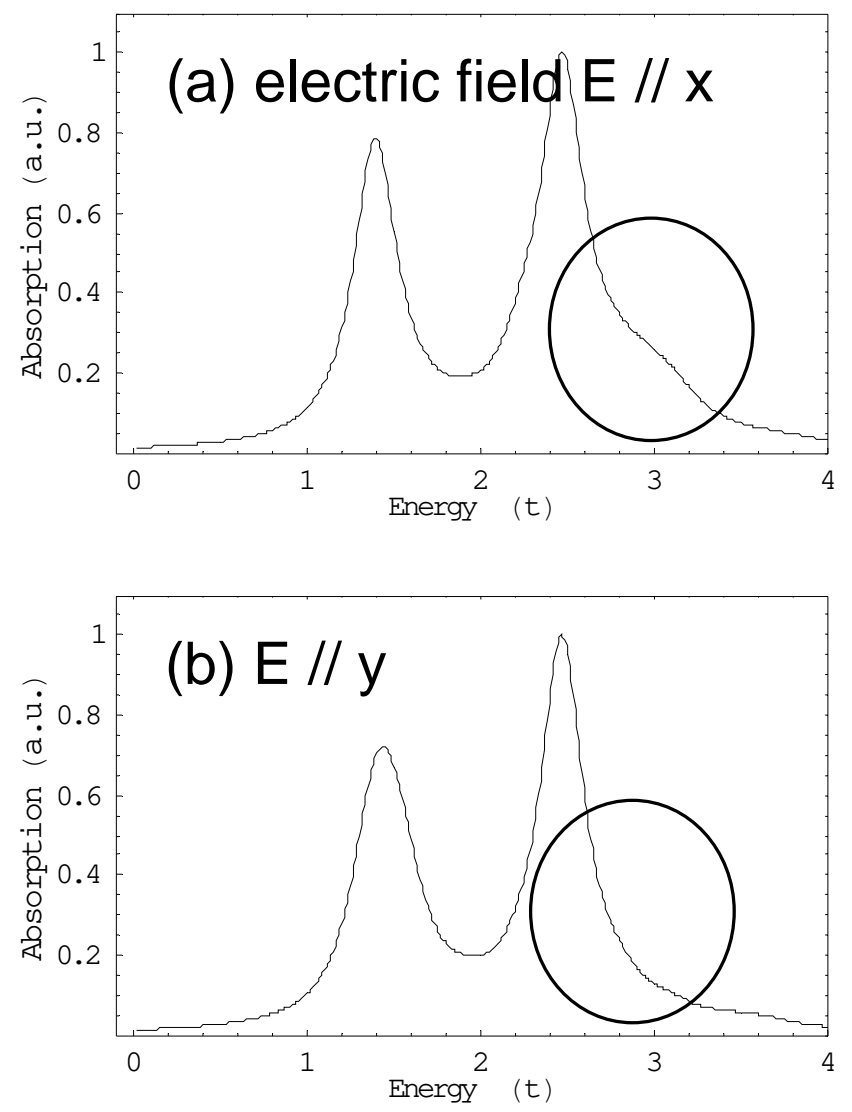

Fig. 6 